Research Article

\title{
Study on Certain Subclass of Analytic Functions Involving Mittag- Leffler Function
}

\author{
Nazek Alessa $\mathbb{D}^{1},{ }^{1}$ B. Venkateswarlu, ${ }^{2}$ K. Loganathan $\mathbb{D}^{\mathbb{D}},{ }^{3,4}$ P. Thirupathi Reddy, ${ }^{5}$ \\ A. Shashikala, ${ }^{2}$ and Ngawang Namgyel ${ }^{6}{ }^{6}$ \\ ${ }^{1}$ Department of Mathematical Sciences, Faculty of Science, Princess Nourah Bint Abdulrahman University, Saudi Arabia \\ ${ }^{2}$ Department of Mathematics, GSS, GITAM University, Doddaballapur, 562 163, Bengaluru Rural, Karnataka, India \\ ${ }^{3}$ Research and Development Wing, Live4Research, Tiruppur, 638106 Tamilnadu, India \\ ${ }^{4}$ Department of Mathematics and Statistics, Manipal University Jaipur, Jaipur, Rajasthan, India \\ ${ }^{5}$ Department of Mathematics, Kakatiya University, Warangal, 506009 Telangana, India \\ ${ }^{6}$ Department of Humanities and Management, Jigme Namgyel Engineering College, Royal University of Bhutan, \\ Dewathang, Bhutan
}

Correspondence should be addressed to K. Loganathan; loganathankaruppusamy304@gmail.com and Ngawang Namgyel; ngawangnamgyel@jnec.edu.bt

Received 24 August 2021; Revised 5 October 2021; Accepted 15 October 2021; Published 28 October 2021

Academic Editor: Huo Tang

Copyright (C) 2021 Nazek Alessa et al. This is an open access article distributed under the Creative Commons Attribution License, which permits unrestricted use, distribution, and reproduction in any medium, provided the original work is properly cited.

We propose and explore a new subclass of regular functions described by a new derivative operator in this paper. Some coefficient estimations, growth and distortion aspects, extreme points, star-like radii, convexity, Fekete-Szego inequality, and partial sums are derived.

\section{Introduction}

Let $\mathscr{A}$ represent the regular function class $u$ defined on the disk $U=\{w:|w|<1\}$ normalized by $u$ (i.e., $u(0)=0$ and $u^{\prime}$ $(0)=1)$. The origin of the form is about the Taylor series expansion of such an equation

$$
u(w)=w+\sum_{\eta=2}^{\infty} a_{\eta} w^{\eta}
$$

$S$ indicates a subclass of $\mathscr{A}$ consists entirely of mappings that are the same as $U$.

For $u \in \mathscr{A}$ presented by (2) and $g(w)$ specified by

$$
g(w)=w+\sum_{\eta=2}^{\infty} b_{\eta} w^{\eta}
$$

their convolution, represented by $(u * g)$, is specified as

$$
(u * g)(w)=w+\sum_{n=2}^{\infty} a_{n} b_{n} w^{n}=(g * u)(w)(w \in U)
$$

The $\mathscr{A}$ subclass consisting of the $u$-type function is specified by $T$ as

$$
u(w)=w-\sum_{\eta=2}^{\infty} a_{\eta} w^{\eta}
$$

Silverman [1] extensively examined this subclass.

The study of operators plays an important role in geometric function theory in complex analysis and its related fields. Many derivative and integral operators can be written in terms of convolution of certain analytic functions. It is observed that this formalism brings an ease in further mathematical exploration and also helps to better understand the geometric properties of such operators. The Mittag-Leffler function $[2,3]$ is defined by the following power series, 
convergent in the whole complex plane:

$$
E_{v}(w):=\sum_{\eta=0}^{\infty} \frac{w^{\eta}}{\Gamma(v \eta+1)}, \quad v>0, w \in \mathbb{C}
$$

We recognize that it is an entire function of order $1 / v$ providing a simple generalization of the exponential function $\exp (w)$ to which it reduces for $v=1$. For detailed information on the Mittag-Leffler-type functions and their laplace transforms, the reader may consult, e.g., [4-6] and the recent treatise by Gorenflo et al. [7].

We also note that for the convergence of the power series in (5), the parameter $v$ may be complex provided that $\mathfrak{R}(v)>0$. The most interesting properties of the Mittag-Leffler function are associated with its asymptotic expansions as $w \longrightarrow \infty$ in various sectors of the complex plane. A more general function $E_{v, \tau}$ generalizing $E_{v}(w)$ was introduced by Wiman [8] and defined by

$$
E_{v, \tau}(w)=\sum_{\eta=0}^{\infty} \frac{w^{\eta}}{\Gamma(v \eta+\tau)}(w, v, \tau \in \mathbb{C}, \mathfrak{R}(v)>0, \mathfrak{R}(\tau)>0)
$$

Observe that the function $E_{v, \tau}$ contains many wellknown functions as its special case, for example, $E_{1,1}(w)=e^{w}, E_{1,2}(w)=\left(e^{w}-1\right) / w, E_{2,1}\left(w^{2}\right)=\cosh w, E_{2,1}(-$ $\left.w^{2}\right)=\cos w, E_{2,2}\left(w^{2}\right)=\sinh w / w, E_{2,2}\left(-w^{2}\right)=\sinh w / w, E_{4}$ $=1 / 2\left[\cos w^{1 / 4}+\cosh w^{1 / 4}\right], \quad$ and $\quad E_{3}=1 / 2\left[e^{w^{1 / 3}}+2 e^{-(1 / 3)}\right.$ $\left.\cos \left((\sqrt{3} / 2) w^{1 / 3}\right)\right]$.

The Mittag-Leffler function arises naturally in the solution of fractional-order differential and integral equations and especially in the investigations of fractional generalization of kinetic equation, random walks, Levy flights, and super diffusive transport and in the study of complex systems. Several properties of Mittag-Leffler function and generalized Mittag-Leffler function can be found, e.g., in [9-16]. Observe that Mittag-Leffler function $E_{v, \tau}(w)$ does not belong to the family $A$. Thus, it is natural to consider the following normalization of Mittag-Leffler functions as below:

$$
E_{v, \tau}=w \Gamma \tau E_{v, \tau}(w)=w+\sum_{\eta=2}^{\infty} \frac{\Gamma(\tau)}{\Gamma(v(\eta-1)+\tau)} w^{\eta}
$$

It holds for complex parameters $v, \tau$ and $w \in \mathbb{C}$. The function $Q_{v, \tau}(w)$ is specified by

$$
Q_{v, \tau}(w)=w \Gamma(\tau) E_{v, \tau}(w)
$$

Now, for $u \in \mathscr{A}$, the derivative operator that follows is defined by $\mathscr{D}_{\hbar}^{m}(v, \tau) u: \mathscr{A} \longrightarrow \mathscr{A}$ by

$$
\begin{gathered}
\mathscr{D}_{\hbar}^{0}(v, \tau) u(w)=u(w) * Q_{v, \tau}(w), \\
\mathscr{D}_{\hbar}^{1}(v, \tau) u(w)=(1-\hbar)\left(u(w) * Q_{v, \tau}(w)\right)+\hbar w\left(u(w) * Q_{v, \tau}(w)\right)^{\prime}, \\
\vdots \\
\mathscr{D}_{\hbar}^{m}(v, \tau) u(w)=\mathscr{D}_{\hbar}^{1}\left(\mathscr{D}_{\hbar}^{m-1}(v, \tau) u(w)\right) .
\end{gathered}
$$

If $u$ is specified by (1), then from the operator's definition $\mathscr{D}_{\hbar}^{m} u$, it is clear to see that

$$
\mathscr{D}_{\hbar}^{m}(v, \tau) u(w)=w+\sum_{\eta=2}^{\infty} \phi_{\eta}^{m}(\hbar, v, \tau) a_{\eta} w^{\eta}
$$

where

$$
\phi_{\eta}^{m}(\hbar, v, \tau)=\frac{\Gamma(\tau)}{\Gamma(v(\eta-1)+\tau)}[\hbar(\eta-1)+1]^{m}
$$

Keep in mind that

(1) the Al-Oboudi operator [17] is achieved when $v=0$ and $\tau=1$

(2) we get the Salagean operator [18] when $v=0, \tau=1$, and $\hbar=1$

(3) when $m=0$, we get $E_{v, \tau}(w)$, according to Srivastava et al. [19]

If $u \in T$ is represented by (4), then we have got it.

$$
\mathscr{D}_{\hbar}^{m}(v, \tau) u(w)=w-\sum_{\eta=2}^{\infty} \phi_{\eta}^{m}(\hbar, v, \tau) a_{\eta} w^{\eta}
$$

Now, by utilizing the differential operator, $\mathscr{D}_{\hbar}^{m}(v, \tau) u$, a new subclass of functions belonging to the class $\mathscr{A}$ is specified.

Definition 1. For $0 \leq v \leq 1, \ell \geq 1, k \geq 0$, and $0 \leq \wp<1$, a mapping in a class is referred to as $u \in \mathscr{A} S_{\hbar, v, \tau}^{m}(\nu, \ell, k, \wp)$, if it satisfies the case

$$
\mathfrak{R}\left\{\ell \frac{w \aleph^{\prime}(w)}{\aleph(w)}-(\ell-1)\right\}>k\left|\ell \frac{w \aleph^{\prime}(w)}{\aleph(w)}-\ell\right|+\wp
$$

where

$$
\aleph(w)=(1-v) \mathscr{D}_{\hbar}^{m} u(w)+v w\left(\mathscr{D}_{\hbar}^{m} u(w)\right)^{\prime} .
$$

We also define $T S_{\hbar, v, \tau}^{m}(\nu, \ell, k, \wp)=S_{\hbar, v, \tau}^{m}(\nu, \ell, k, \wp) \cap T$.

For special situations of characteristics, $S_{\hbar, v, \tau}^{m}(\nu, \ell, k, \wp)$ and $T S_{\hbar, v, \tau}^{m}(\nu, \ell, k, \wp)$, it can be reduced to new or known categories of functions studied in recent research [20-25].

The objective of this review is to look into a variety of properties for functions in the aforesaid class. For specific parameter instances. 


\section{Coefficient Estimates}

To get our results, we will require the subsequent lemma.

Lemma 2 (see [26]). Let $\wp$ be a real and $z$ be a complex number. Then, $\mathfrak{R}(z) \geq \wp$.

$$
\Longleftrightarrow|z+(1-\wp)|-|z-(1+\wp)| \geq 0 .
$$

For beginnings, we have a coefficient that is relevant for functions in the classs $S_{\hbar, v, \tau}^{m}(\nu, \ell, k, \wp)$.

Theorem 3. Let $u \in \mathscr{A}$ indicated by (1). If

$$
\sum_{\eta=2}^{\infty}[1-\wp+\ell(\eta-1)(1+k)] \chi_{\eta}(\hbar, v, m, v, \tau)\left|a_{\eta}\right| \leq 1-\wp,
$$

where

$$
\chi_{\eta}(\hbar, v, m, v, \tau)=[1+v(m-1)] \phi_{\eta}^{m}(\hbar, v, \tau)
$$

then $u \in S_{\hbar, v, \tau}^{m}(\nu, \ell, k, \wp)$.

Proof. In the definition by consequence of 1 and Lemma 2, it is enough to demonstrate that

$$
\begin{aligned}
& \left|\ell \frac{w \aleph^{\prime}(w)}{\aleph(w)}-(\ell-1)-k\right| \ell \frac{w \aleph^{\prime}(w)}{\aleph(w)}-\ell|-(1+\wp)| \\
& \quad \leq\left|\ell \frac{w \aleph^{\prime}(w)}{\aleph(w)}-(\ell-1)-k\right| \ell \frac{w \aleph^{\prime}(w)}{\aleph(w)}-\ell|+(1+\wp)| .
\end{aligned}
$$

For the R.H.S and L.H.S of (18), we may, respectively, write

$$
\begin{aligned}
R & =\left|\ell \frac{w \aleph^{\prime}(w)}{\aleph(w)}-(\ell-1)-k\right| \ell \frac{w \aleph^{\prime}(w)}{\aleph(w)}-\ell|+(1-\wp)| \\
& =\frac{1}{|\aleph(w)|}\left|\ell w \aleph^{\prime}(w)-(\ell-1) \aleph(w)-k e^{i \theta}\right| \ell w \aleph^{\prime}(w)-\ell \aleph(w)|+(1-\wp) \aleph(w)| \\
& >\frac{|w|}{|\aleph(w)|}\left[2-\wp-\sum_{\eta=2}^{\infty}[2-\wp+\ell(\eta-1)(k+1)]\right] \chi_{\eta}(\hbar, v, m, v, \tau)\left|a_{\eta}\right|,
\end{aligned}
$$

and similarly,

$$
\begin{aligned}
L & =\left|\ell \frac{w \aleph^{\prime}(w)}{\aleph(w)}-(\ell-1)-k\right| \ell \frac{w \aleph^{\prime}(w)}{\aleph(w)}-\ell|-(1+\wp)| \\
& =\frac{1}{|\aleph(w)|}\left|\ell w \aleph^{\prime}(w)-(\ell-1) \aleph(w)-k e^{i \theta}\right| \ell w \aleph^{\prime}(w)-\ell \aleph(w)|-(1+\wp) \aleph(w)| \\
& <\frac{|w|}{|\aleph(w)|}\left[\wp+\sum_{\eta=2}^{\infty}[\ell(\eta-1)(1+k)-\wp]\right] \chi_{\eta}(\hbar, v, m, v, \tau)\left|a_{\eta}\right| .
\end{aligned}
$$

Then,

$R-L>\frac{|w|}{|\aleph(w)|}\left[2(1-\wp)-2 \sum_{\eta=2}^{\infty}[1-\wp+\ell(\eta-1)(1+k)] \chi_{\eta}(\hbar, v, m, v, \tau)\left|a_{\eta}\right|\right] \geq 0$.

The condition (16) required is fulfilled.

We have a necessary and adequate situation in the next theorem for a function $u \in T$ to be in the class $T$ $S_{\hbar, v, \tau}^{m}(\nu, \ell, k, \wp)$.

Theorem 4. Let $u \in T$ indicated by (3). Then, $u \in T S_{\hbar, v, \tau}^{m}$ $(v, \ell, k, \wp)$.

$$
\Longleftrightarrow \sum_{\eta=2}^{\infty}[1-\wp+\ell(\eta-1)(1+k)] \chi_{\eta}(\hbar, v, m, v, \tau) a_{\eta} \leq 1-\wp,
$$

where $\chi_{\eta}(\hbar, v, m, v, \tau)$ is defined by (17).

Proof. We can only prove the requirement in view 3 of the theorem. If $u \in T S_{\hbar, v, \tau}^{m}(\nu, \ell, k, \wp)$ and $w$ is real, then

$$
\begin{gathered}
\frac{1-\sum_{\eta=2}^{\infty}[1+\ell(\eta-1)] \chi_{\eta}(\hbar, v, m, v, \tau) a_{\eta} w^{\eta-1}}{1-\sum_{\eta=2}^{\infty} \chi_{\eta}(\hbar, v, m, v, \tau) a_{\eta} w^{\eta-1}}-\wp \\
>k\left|\frac{\sum_{\eta=2}^{\infty} \ell(\eta-1) \chi_{\eta}(\hbar, v, m, v, \tau) a_{\eta} w^{\eta-1}}{1-\sum_{\eta=2}^{\infty} \chi_{\eta}(\hbar, v, m, v, \tau) a_{\eta} w^{\eta-1}}\right| .
\end{gathered}
$$

We get the desired inequality from letting $w \longrightarrow 1^{-}$.

Corollary 5. If $u \in T S_{\hbar, v, \tau}^{m}(\nu, \ell, k, \wp)$, then

$$
a_{\eta} \leq \frac{1-\wp}{[1-\wp+\ell(\eta-1)(1+k)] \chi_{\eta}(\hbar, v, m, v, \tau)}(\eta \geq 2) .
$$

\section{Growth and Distortion Theorem}

Theorem 6. Let $u \in T S_{\hbar, v, \tau}^{m}(\nu, \ell, k, \wp)$. Then, for $|w|=r<1$,

$$
r-\frac{(1-\wp)}{B_{2}(\hbar, v, m, v, \tau, \ell, k, \wp)} r^{2} \leq|u(w)| \leq r+\frac{(1-\wp)}{B_{2}(\hbar, v, m, v, \tau, \ell, k, \wp)} r^{2},
$$

$$
1-\frac{2(1-\wp)}{B_{2}(\hbar, v, m, v, \tau, \ell, k, \wp)} r^{2} \leq\left|u^{\prime}(w)\right| \leq 1+\frac{2(1-\wp)}{B_{2}(\hbar, v, m, v, \tau, \ell, k, \wp)} r,
$$

where

$$
B_{\eta}(\hbar, v, m, v, \tau, \ell, k, \wp)=[1-\wp+\ell(\eta-1)(1+k)] \chi_{\eta}(\hbar, v, m, v, \tau)(\eta \geq 2)
$$


Equations (25) and (26) are sharp for the u given function

$$
u(w)=w-\frac{(1-\wp)}{B_{2}(\hbar, v, m, v, \tau, \ell, k, \wp)} w^{2} .
$$

Proof. Since $u \in T S_{\hbar, v, \tau}^{m}(\nu, \ell, k, \wp)$ and it follows from 4 of the theorem,

$$
\sum_{\eta=2}^{\infty} B_{\eta}(\hbar, v, m, v, \tau, \ell, k, \wp) a_{\eta} \leq(1-\wp),
$$

where $B_{\eta}(\hbar, v, m, v, \tau, \ell, k, \wp)$ is given by (27), we have

$$
\begin{aligned}
B_{2}(\hbar, v, m, v, \tau, \ell, k, \wp) \sum_{\eta=2}^{\infty} a_{\eta} & =\sum_{\eta=2}^{\infty} B_{2}(\hbar, v, m, v, \tau, \ell, k, \wp) a_{\eta} \\
& \leq \sum_{\eta=2}^{\infty} B_{\eta}(\hbar, v, m, v, \tau, \ell, k, \wp) a_{\eta} \\
& \leq 1-\wp,
\end{aligned}
$$

and therefore,

$$
\sum_{\eta=2}^{\infty} a_{\eta} \leq \frac{(1-\wp)}{B_{2}(\hbar, v, m, v, \tau, \ell, k, \wp)} .
$$

Since $u$ is given by (3), we get

$$
\begin{gathered}
|u(w)| \leq|w|+|w|^{2} \sum_{\eta=2}^{\infty} a_{\eta}|w|^{\eta-2} \leq r+r^{2} \sum_{\eta=2}^{\infty} a_{\eta} \\
\leq r+\frac{(1-\wp)}{B_{2}(\hbar, v, m, v, \tau, \ell, k, \wp)} r^{2} \\
|u(w)| \geq|w|-|w|^{2} \sum_{\eta=2}^{\infty} a_{\eta}|w|^{\eta-2} \geq r-r^{2} \sum_{\eta=2}^{\infty} a_{\eta} \\
\geq r-\frac{(1-\wp)}{B_{2}(\hbar, v, m, v, \tau, \ell, k, \wp)} r^{2} .
\end{gathered}
$$

In light of Theorem 4, we have

$$
\begin{aligned}
\frac{B_{2}(\hbar, v, m, v, \tau, \ell, k, \wp)}{2} \sum_{\eta=2}^{\infty} \eta a_{\eta} & =\sum_{\eta=2}^{\infty} \frac{B_{2}(\hbar, v, m, v, \tau, \ell, k, \wp)}{2} \eta a_{\eta} \\
& \leq \sum_{\eta=2}^{\infty} B_{\eta}(\hbar, v, m, v, \tau, \ell, k, \wp) a_{\eta} \leq(1-\wp),
\end{aligned}
$$

which yields

$$
\sum_{\eta=2}^{\infty} \eta a_{\eta} \leq \frac{2(1-\wp)}{B_{2}(\hbar, v, m, v, \tau, \ell, k, \wp)}
$$

Thus,

$$
\begin{aligned}
\left|u^{\prime}(w)\right| & \leq 1+\sum_{\eta=2}^{\infty} \eta a_{\eta}|w|^{\eta-1} \leq 1+r \sum_{\eta=2}^{\infty} \eta a_{\eta} \\
& \leq 1+\frac{2(1-\wp)}{B_{2}(\hbar, v, m, v, \tau, \ell, k, \wp)} r, \\
\left|u^{\prime}(w)\right| & \geq 1-\sum_{\eta=2}^{\infty} \eta a_{\eta}|w|^{\eta-1} \geq 1-r \sum_{\eta=2}^{\infty} \eta a_{\eta} \\
& \geq 1-\frac{2(1-\wp)}{B_{2}(\hbar, v, m, v, \tau, \ell, k, \wp)} r .
\end{aligned}
$$

Hence, the proof is complete.

Consider that $|u(w)|=\mid w-\left((1-\wp) /\left(B_{2}(\hbar, v, m, v, \tau, \ell, k, \wp)\right)\right.$ $\left.B_{2}(\hbar, v, m, v, \tau, \ell, k, \wp)\right) w^{2} \mid$ is sharp is to (25).

And $\left|u^{\prime}(w)\right|=\mid 1-\left((2(1-\wp)) 2(1-\wp) /\left(B_{2}(\hbar, v, m, v, \tau, \ell\right.\right.$, $\left.k, \wp)) B_{2}(\hbar, v, m, v, \tau, \ell, k, \wp)\right) w \mid$ is sharp is to $(26)$.

\section{Extreme Points}

Now, for the function class, we look at the extreme points $T S_{\hbar, v, \tau}^{m}(\nu, \ell, k, \wp)$.

Theorem 7. Let the functions $u_{1}(w)=w$ and

$$
u_{\eta}(w)=w-\frac{(1-\wp)}{B_{\eta}(\hbar, v, m, v, \tau, \ell, k, \wp)} w^{\eta}(\eta \geq 2) .
$$

Then, $u \in T S_{\hbar, v, \tau}^{m}(\nu, \ell, k, \wp)$.

$$
\Longleftrightarrow u(w)=\sum_{\eta=2}^{\infty} \hbar_{\eta} u_{\eta}(w)(w \in U),
$$

where $\hbar_{\eta} \geq 0(\eta \geq 1)$ and $\sum_{\eta=1}^{\infty} \hbar_{\eta}=1$.

Proof. Assume that it is possible to write $u$ as in (37). Then,

$$
\begin{aligned}
u(w) & =\hbar_{1} w+\sum_{\eta=2}^{\infty} \hbar_{\eta}\left[w-\frac{(1-\wp)}{B_{\eta}(\hbar, v, m, v, \tau, \ell, k, \wp)} w^{\eta}\right] \\
& =w-\sum_{\eta=2}^{\infty} \hbar_{\eta} \frac{(1-\wp)}{B_{\eta}(\hbar, v, m, \ell, k, \wp)} w^{\eta},
\end{aligned}
$$

since

$$
\begin{aligned}
& \sum_{\eta=2}^{\infty} B_{\eta}(\hbar, v, m, v, \tau, \ell, k, \wp) \hbar_{\eta} \frac{(1-\wp)}{B_{\eta}(\hbar, v, m, v, \tau, \ell, k, \wp)} \\
& =(1-\wp) \sum_{\eta=2}^{\infty} \hbar_{\eta}=(1-\wp)\left(1-\hbar_{1}\right) \leq(1-\wp) .
\end{aligned}
$$

By virtue 4 of the theorem, it follows that $u \in T S_{\hbar, v, \tau}^{m}$ $(\nu, \ell, k, \wp)$. 
Conversely, suppose $u \in T S_{\hbar, v, \tau}^{m}(\nu, \ell, k, \wp)$ and consider

$$
\begin{gathered}
\hbar_{\eta}=\frac{B_{\eta}(\hbar, v, m, v, \tau, \ell, k, \wp)}{(1-\wp)} a_{\eta}, \quad \eta \geq 2, \\
\hbar_{1}=1-\sum_{\eta=2}^{\infty} \hbar_{\eta} .
\end{gathered}
$$

Then, $u(w)=\sum_{\eta=1}^{\infty} \hbar_{\eta} u_{n}(w)$, hence the theorem.

\section{Radii of Starlikeness, Convexity, and Close- to-Convexity}

Theorem 8. Let $u \in T S_{\hbar, v, \tau}^{m}(v, \ell, k, \wp)$. Then, $u$ is star-shaped of order $\rho(0 \leq \rho<1)$ in $|w|<r_{1}(\hbar, \nu, m, v, \tau, \ell, k, \wp)$, where

$r_{1}(\hbar, v, m, v, \tau, \ell, k, \wp)=\inf _{\eta \geq 2}\left[\frac{(1-\rho) B_{\eta}(\hbar, v, m, v, \tau, \ell, k, \wp)}{(\eta-\rho)(1-\wp)}\right]^{1 /(\eta-1)}$.

Proof. To be able to prove the theorem, we have to demonstrate that

$$
\left|\frac{w u^{\prime}(w)}{u(w)}-1\right| \leq 1-\rho
$$

$0 \leq \rho<1$ for $w \in U$ with $|w|<r_{1}(\hbar, \nu, m, v, \tau, \ell, k, \wp)$. We have

$\left|\frac{w u^{\prime}(w)}{u(w)}-1\right|=\left|\frac{-\sum_{\eta=2}^{\infty}(\eta-1) a_{\eta} w^{\eta-1}}{1-\sum_{\eta=2}^{\infty} a_{\eta} w^{\eta-1}}\right| \leq \frac{\sum_{\eta=2}^{\infty}(\eta-1) a_{\eta}|w|^{\eta-1}}{1-\sum_{\eta=2}^{\infty} a_{\eta}|w|^{\eta-1}}$.

Thus,

$$
\left|\frac{w u^{\prime}(w)}{u(w)}-1\right| \leq 1-\rho \quad \text { if } \sum_{\eta=2}^{\infty} \frac{(\eta-\rho)}{(1-\rho)} a_{\eta}|w|^{\eta-1} \leq 1 .
$$

In virtue of (22), we have

$$
\frac{\sum_{\eta=2}^{\infty} B_{\eta}(\hbar, v, m, v, \tau, \ell, k, \wp)}{1-\wp} a_{\eta} \leq 1 .
$$

The inequality of (43) would then be valid if

$$
\frac{(\eta-\rho)}{(1-\rho)}|w|^{\eta-1} \leq \frac{B_{\eta}(\hbar, v, m, v, \tau, \ell, k, \wp)}{1-\wp}(\eta \geq 2)
$$

or if

$$
|w| \leq\left[\frac{(1-\rho) B_{\eta}(\hbar, v, m, v, \tau, \ell, k, \wp)}{(\eta-\rho)(1-\wp)}\right]^{1 /(\eta-1)}(\eta \geq 2) .
$$

Hence, the proof is complete.

The evidence 9 and 10 of the subsequent theorems is comparable to 8 of the theorem, so the evidence is excluded.

Theorem 9. Let $u \in T S_{\hbar, v, \tau}^{m}(\nu, \ell, k, \wp)$. Then, $u$ is convex of order $\rho(0 \leq \rho<1)$ in $|w|<r_{2}(\hbar, v, m, v, \tau, \ell, k, \wp)$, where

$r_{2}(\hbar, v, m, v, \tau, \ell, k, \wp)=\inf _{\eta \geq 2}\left[\frac{(1-\rho) B_{\eta}(\hbar, v, m, v, \tau, \ell, k, \wp)}{\eta(\eta-\rho)(1-\wp)}\right]^{1 /(\eta-1)}$.

Theorem 10. Let the function $u$ given by (3) be in the class $T S_{\hbar, v, \tau}^{m}(\nu, \ell, k, \wp)$. Then, $u$ in close-to-convex of order $\rho(0 \leq \rho$ $<1)$ in $|w|<r_{3}(\hbar, v, m, v, \tau, \ell, k, \wp)$, where

$r_{3}(\hbar, v, m, v, \tau, \ell, k, \wp)=\inf _{\eta \geq 2}\left[\frac{(1-\rho) B_{\eta}(\hbar, v, m, v, \tau, \ell, k, \wp)}{\eta(1-\wp)}\right]^{1 /(\eta-1)}$.

\section{Fekete-Szego Inequality}

In this section, for the mapping in the class, we get the Fekete-Szego inequality $S_{\hbar, v, \tau}^{m}(\nu, \ell, k, \wp)$. To illustrate our fundamental result, we will identify the appropriate lemma.

Lemma 11 (see [27]). If $p(w)=1+c_{1} w+c_{2} w+c_{3} w^{2}+\cdots$ is an analytic mapping with positive real part in $U$, then

$$
\left|c_{2}-\jmath c_{1}^{2}\right|= \begin{cases}-4 \jmath+2, & \jmath \leq 0, \\ 2, & 0 \leq \jmath \leq 1, \\ 4 \jmath-2, & \jmath \geq 1 .\end{cases}
$$

When $j<0$ or $j>1$, the inequality holds iff $p(w)=(1+$ $w) /(1-w)$ or one of its rotations. If $0<j<1$, then the equality holds iff

$$
p(w)=\frac{1+w^{2}}{1-w^{2}}
$$

or one of its rotations. If $\mathrm{J}=0$, the equality holds iff

$$
p(w)=\left(\frac{1+\delta}{2}\right) \frac{1+w}{1-w}+\left(\frac{1-\delta}{2}\right) \frac{1-w}{1+w}(0 \leq \delta \leq 1)
$$

or one of its rotations.

If $J=1$, the equality holds iff $p(w)$ is the reciprocal of one of the mapping such that the equality holds when it comes to $J=0$. 
Theorem 12. Let $\ell \geq 1,0 \leq k \leq \wp<1$. If $u \in S_{\hbar, v, \tau}^{m}(\nu, \ell, k, \wp)$ is given by (1), then

$$
\left|a_{3}-\mu a_{2}^{2}\right|= \begin{cases}\frac{(1-\wp)}{\ell^{2}(1-k)^{2} \chi_{3}(\hbar, v, m, v, \tau)}\left[\ell(1-k)+2(1-\wp)-4 \mu(1-\wp) \frac{\chi_{3}(\hbar, v, m, v, \tau)}{\chi_{2}^{2}(\hbar, v, m, v, \tau)}\right], & \mu \leq \sigma_{1}, \\ \frac{(1-\wp)}{\ell(1-k) \chi_{3}(\hbar, v, m, v, \tau)}, & \sigma_{1} \leq \mu \leq \sigma_{2}, \\ \frac{-(1-\wp)}{\ell^{2}(1-k)^{2} \chi_{3}(\hbar, v, m, v, \tau)}\left[\ell(1-k)+2(1-\wp)-4 \mu(1-\wp) \frac{\chi_{3}(\hbar, v, m, v, \tau)}{\chi_{2}^{2}(\hbar, v, m, v, \tau)}\right], & \mu \geq \sigma_{2},\end{cases}
$$

where

$$
\begin{aligned}
\sigma_{1} & =\frac{\chi_{2}^{2}(\hbar, v, m, v, \tau)}{2 \chi_{3}(\hbar, v, m, v, \tau)}, \\
\sigma_{2} & =\frac{\chi_{2}^{2}(\hbar, v, m, v, \tau)[1-\wp+\ell(1-k)]}{2 \chi_{3}(\hbar, v, m, v, \tau)(1-\wp)} .
\end{aligned}
$$

The outcome is sharp.

Proof. Since, for complex numbers, $\mathfrak{R}(z) \leq|z|, \quad u \in S_{\hbar, v, \tau}^{m}$ $(\nu, \ell, k, \wp)$ implies that

$$
\mathfrak{R}\left[\ell \frac{w \aleph^{\prime}(w)}{\aleph(w)}-(\ell-1)\right]>k \Re\left[\ell \frac{w \aleph^{\prime}(w)}{\aleph(w)}-\ell\right]+\wp
$$

or that

$$
\mathfrak{R}\left[\frac{w \aleph^{\prime}(w)}{\aleph(w)}\right]>\frac{\wp-1+\ell(1-k)}{\ell(1-k)} .
$$

Hence,

$$
\aleph \in S^{*}\left(\frac{\wp-1+\ell(1-k)}{\ell(1-k)}\right) \text {. }
$$

Let

$$
p(w)=\frac{w \aleph^{\prime}(w) / \aleph(w)-((\wp-1+\ell(1-k) / \ell(1-k))}{(1-\wp) /(\ell(1-k))}=1+c_{1} w+c_{2} w^{2}+\cdots .
$$

We then have, by way of (10) and (14),

$$
\begin{aligned}
& a_{2}=\frac{(1-\wp)}{\ell(1-k) \chi_{2}(\hbar, v, m, v, \tau)} c_{1}, \\
& a_{3}=\frac{(1-\wp)}{2 \ell(1-k) \chi_{2}(\hbar, v, m, v, \tau)}\left[c_{2}+\frac{1-\wp}{\ell(1-k)} c_{1}^{2}\right] .
\end{aligned}
$$

Therefore, we obtain

$$
\begin{aligned}
a_{3}-\mu a_{2}^{2}= & \frac{(1-\wp)}{2 \ell(1-k) \chi_{3}(\hbar, v, m, v, \tau)}\left[c_{2}+\frac{1-\wp}{\ell(1-k)} c_{1}^{2}\right] \\
& -\mu \frac{(1-\wp)^{2}}{\ell^{2}(1-k)^{2} \chi_{2}^{2}(\hbar, v, m, v, \tau)} c_{1}^{2} \\
= & \frac{(1-\wp)}{2 \ell(1-k) \chi_{3}(\hbar, v, m, v, \tau)} \\
& \cdot\left[c_{2}-\frac{1-\wp}{\ell(1-k)} c_{1}^{2}\left(2 \mu \frac{\chi_{3}(\hbar, v, m, v, \tau)}{A_{1}^{2}(\hbar, v, m, v, \tau)}-1\right)\right] .
\end{aligned}
$$

We write

$$
a_{3}-\mu a_{2}^{2}=\frac{(1-\wp)}{2 \ell(1-k) \chi_{3}(\hbar, v, m, v, \tau)}\left(c_{2}-\rho c_{1}^{2}\right),
$$

where

$$
\rho=\frac{(1-\wp)}{\ell(1-k)}\left[2 \mu \frac{\chi_{3}(\hbar, v, m, v, \tau)}{\chi_{2}^{2}(\hbar, v, m, v, \tau)}-1\right] .
$$

The implementation of the lemma above follows our conclusion. Denote

$$
\xi=\frac{\wp-1+\ell(1-k)}{\ell(1-k)} .
$$

If $\mu<\sigma_{1}$ or $\mu>\sigma_{2}$, it is true that equality exists.

$$
\Longleftrightarrow \aleph(w)=\frac{w}{\left(1-e^{i \theta} w\right)^{2(1-\xi)}}(\theta \in \mathbb{R}) .
$$

When $\sigma_{1}<\mu<\sigma_{2}$, it is true that equality exists, iff

$$
\aleph(w)=\frac{w}{\left(1-e^{i \theta} w^{2}\right)^{(1-\xi)}}(\theta \in \mathbb{R}) .
$$


If $\mu=\sigma_{1}$, then it is true that equality exists, iff

$$
\begin{aligned}
\aleph(w) & =\left[\frac{w}{\left(1-e^{i \theta} w\right)^{2(1-\xi)}}\right]^{(1+\delta) / 2}\left[\frac{w}{\left(1+e^{i \theta} w\right)^{2(1-\xi)}}\right]^{(1-\delta) / 2} \\
& =\frac{w}{\left[\left(1-e^{i \theta} w\right)^{1+\delta}\left(1+e^{i \theta} w\right)^{1-\delta}\right]^{1-\xi}}, \quad 0 \leq \delta \leq 1, \theta \in \mathbb{R} .
\end{aligned}
$$

Finally, if it is true that equality exists $\Longleftrightarrow p(w) \mu$ $=\sigma 2$, it is the inverse of one of the equality functions and holds true in the case of $\mu=\sigma 2$

\section{Partial Sums}

Consider the recent works on partial analytic function sums by Silverman [28] and Silvia [29]. Partial function in this class is considered in this section to be $T S_{\hbar,, \tau}^{m}(\nu, \ell, k, \wp)$ giving sharp lower boundaries to the reap part ratios of $u(w)$ to $u q(w)$ and $u^{\prime}(w)$ to $u^{\prime} q(w)$.

Theorem 13. Let $u \in T S_{\hbar, v, \tau}^{m}(\nu, \ell, k, \wp)$ and indicate $u_{1}(w)$ and $u_{q}(w)$ as partial sums

$$
\begin{gathered}
u_{1}(w)=w, \\
u_{q}(w)=w+\sum_{\eta=2}^{q} a_{\eta} w^{\eta}(q \in \mathbb{N} \backslash\{1\}) .
\end{gathered}
$$

Suppose that

$$
\sum_{\eta=2}^{\infty} d_{\eta}\left|a_{\eta}\right| \leq 1
$$

where

$$
d_{\eta}=\frac{[1-\wp+\ell(\eta-1)(1+k)] A_{\eta}(\hbar, v, m, v, \tau)}{1-\wp} .
$$

Then, $u \in T S_{\hbar, v, \tau}^{m}(\nu, \ell, k, \wp)$.

Furthermore,

$$
\begin{aligned}
& \mathfrak{R}\left[\frac{u(w)}{u_{q}(w)}\right]>1-\frac{1}{d_{q+1}}(w \in U, q \in \mathbb{N}), \\
& \mathfrak{R}\left[\frac{u_{q}(w)}{u(w)}\right]>\frac{d_{q+1}}{1+d_{q+1}} .
\end{aligned}
$$

Proof. It is not crucial to verify that the $d_{\eta}$ coefficients supplied by (69) are correct.

$$
d_{\eta+1}>d_{\eta}>1
$$

So we have

$$
\sum_{\eta=2}^{q}\left|a_{\eta}\right|+d_{q+1} \sum_{\eta=q+1}^{\infty}\left|a_{\eta}\right| \leq \sum_{\eta=2}^{\infty} d_{\eta}\left|a_{\eta}\right| \leq 1
$$

The hypothesis used (69), by setting

$$
g_{1}(w)=d_{q+1}\left[\frac{u(w)}{u_{q}(w)}-\left(1-\frac{1}{d_{q+1}}\right)\right]=1+\frac{d_{q+1} \sum_{\eta=q+1}^{\infty} a_{\eta} w^{\eta-1}}{1+\sum_{\eta=2}^{q} a_{\eta} w^{\eta-1}} .
$$

If we use and apply (73), we find that

$$
\left|\frac{g_{2}(w)-1}{g_{2}(w)+1}\right| \leq \frac{d_{q+1} \sum_{\eta=q+1}^{\infty}\left|a_{\eta}\right|}{2-2 \sum_{\eta=2}^{q}\left|a_{\eta}\right|-d_{q+1} \sum_{\eta=q+1}^{\infty}\left|a_{\eta}\right|} \leq 1 .
$$

That immediately leads in a conclusion (70) of Theorem 13. To find out that

$$
u(w)=w+\frac{w^{q+1}}{d_{q+1}}
$$

gives sharp result, we observe that for $w=r e^{i \pi / q}$,

$$
\frac{u(w)}{u_{q}(w)}=1+\frac{w^{q}}{d_{q+1}} \longrightarrow 1-\frac{1}{d_{q+1}} \quad \text { as } w \longrightarrow 1^{-} .
$$

Similarly, if we take

$$
\begin{aligned}
g_{2}(w) & =\left(1+d_{q+1}\right)\left(\frac{u_{q}(w)}{u(w)}-\frac{d_{q+1}}{1+d_{q+1}}\right) \\
& =1-\frac{\left(1+d_{\eta+1}\right) \sum_{\eta=q+1}^{\infty} a_{\eta} w^{\eta-1}}{1+\sum_{\eta=2}^{\infty} a_{\eta} w^{\eta-1}},
\end{aligned}
$$

we can deduce, and make use of (73), that

$$
\left|\frac{g_{2}(w)-1}{g_{2}(w)+1}\right| \leq \frac{\left(1+d_{q+1}\right) \sum_{\eta=q+1}^{\infty}\left|a_{\eta}\right|}{2-2 \sum_{\eta=2}^{q}\left|a_{\eta}\right|-\left(1-d_{q+1}\right) \sum_{\eta=q+1}^{\infty}\left|a_{\eta}\right|} .
$$

This leads directly to the statement (71) of Theorem 13 .

For each $q \in \mathbb{N}$ with the external mapping $u(w)$, the bound in (71) is sharp indicated by (76).

Thus, the evidence of the Theorem 13 is complete.

Theorem 14. Let $u \in T S_{\hbar, v, \tau}^{m}(\nu, \ell, k, \wp)$ and fulfill (16). Then,

$$
\Re\left[\frac{u^{\prime}(w)}{u_{q}^{\prime}(w)}\right] \geq 1-\frac{q+1}{d_{q+1}} .
$$


Proof. By setting

$$
\begin{aligned}
g(w) & =d_{q+1}\left[\frac{u^{\prime}(w)}{u_{q}^{\prime}(w)}\right]-\left(1-\frac{q+1}{d_{q+1}}\right) \\
& =\frac{1+\left(d_{q+1} /(q+1) q+1\right) \sum_{\eta=q+1}^{\infty} n a_{\eta} w^{\eta-1}+\sum_{\eta=2}^{\infty} \eta a_{\eta} w^{\eta-1}}{1+\sum_{\eta=2}^{\infty} \eta a_{\eta} w^{\eta-1}} \\
& =1+\frac{\left(d_{q+1} /(q+1) q+1\right) \sum_{\eta=q+1}^{\infty} \eta a_{\eta} w^{\eta-1}}{1+\sum_{\eta=2}^{\infty} \eta a_{\eta} w^{\eta-1}} .
\end{aligned}
$$

Now,

$\left|\frac{g(w)-1}{g(w)+1}\right| \leq \frac{\left(d_{q+1} /(q+1) q+1\right) \sum_{\eta=q+1}^{\infty} \eta\left|a_{\eta}\right|}{2-2 \sum_{\eta=2}^{q} \eta\left|a_{\eta}\right|-\left(d_{q+1} /(q+1) q+1\right) \sum_{\eta=q+1}^{\infty} \eta\left|a_{\eta}\right|}$

Now,

$$
\left|\frac{g(w)-1}{g(w)+1}\right| \leq 1 \quad \text { if } \sum_{\eta=2}^{q} \eta\left|a_{\eta}\right|+\frac{d_{q+1}}{q+1} \sum_{\eta=q+1}^{\infty} \eta\left|a_{\eta}\right| \leq 1
$$

since the L.H.S. of (83) is bounded above by $\sum_{\eta=2}^{q} d_{\eta}\left|a_{\eta}\right|$ if

$$
\sum_{\eta=2}^{q}\left(d_{\eta}-\eta\right)\left|a_{\eta}\right|+\sum_{\eta=q+1}^{\infty} d_{\eta}-\frac{d_{q+1}}{q+1} \eta\left|a_{\eta}\right| \geq 0
$$

and the proof is complete.

The consequence of the extreme function is sharp $u(w)$ $=w+w^{q+1} / d_{q+1}$.

Theorem 15. Let $u \in T S_{\hbar, v, \tau}^{m}(\nu, \ell, k, \wp)$ and fulfill (16). Then,

$$
\mathfrak{R}\left[\frac{u_{q}^{\prime}(w)}{u^{\prime}(w)}\right] \geq \frac{d_{q+1}}{q+1+d_{q+1}} .
$$

Proof. By setting

$$
\begin{aligned}
g(w) & =\left[q+1+d_{q+1}\right]\left[\frac{u_{q}^{\prime}(w)}{u^{\prime}(w)}-\frac{d_{q+1}}{q+1+d_{q+1}}\right] \\
& =1-\frac{\left(1+\left(d_{q+1} /(q+1) q+1\right)\right) \sum_{\eta=q+1}^{\infty} \eta a_{\eta} w^{\eta-1}}{1+\sum_{\eta=2}^{q} \eta a_{\eta} w^{\eta-1}} .
\end{aligned}
$$

Using (84) and making use of it, we deduce that

$$
\left|\frac{g(w)-1}{g(w)+1}\right| \leq \frac{\left(1+\left(d_{q+1} /(q+1) q+1\right)\right) \sum_{\eta=q+1}^{\infty} \eta\left|a_{\eta}\right|}{2-2 \sum_{\eta=2}^{q} \eta\left|a_{\eta}\right|-\left(1+\left(d_{q+1} /(q+1) q+1\right)\right) \sum_{\eta=q+1}^{\infty} \eta\left|a_{\eta}\right|} \leq 1
$$

that immediately leads us to the statement 15 of the theorem.

\section{Conclusions}

This research has introduced study a new differential operator related to analytic function and studied some basic properties of geometric function theory. Accordingly, some results to coefficient estimates, grouth and distortion theorem, Fekete-Szego inequalityy, and partial sums have also been considered, inviting future research for this field of study.

\section{Data Availability}

No data were used to find this study.

\section{Conflicts of Interest}

The authors declare that they have no conflicts of interest.

\section{Authors' Contributions}

This work was equally contributed by all writers. The final version of the work has been read and approved by all of the authors.

\section{Acknowledgments}

This research was funded by the Deanship of Scientific Research at Princess Nourah Bint Abdulrahman University through the Fast-track Research Funding Program.

\section{References}

[1] H. Silverman, "Univalent functions with negative coefficients," Proceedings of the American Mathematical Society, vol. 51, no. 1, pp. 109-116, 1975.

[2] G. M. Mittag-Leffler, "Sur la nouvelle fonction E (x)," Comptes rendus de l'Académie des Sciences, vol. 137, no. 2, pp. 554-558, 1903.

[3] G. M. Mittag-Leffler, "Sur la représentation analytique d'une branche uniforme d'une fonction monogène: cinquième note," Acta Mathematica, vol. 29, pp. 101-181, 1905.

[4] A. Erdlyi, W. Magnus, F. Oberhettinger, and F. Tricomi, Higher Transcendental Functions, vol. 3, McGraw-Hill, New York, NY, USA, 1955.

[5] R. Gorenflo and F. Mainardi, "Fractional calculus: integral and differential equations of fractional order," in Fractals and Fractional Calculus in Continuum Mechanics, A. Carpinteri and F. Mainardi, Eds., pp. 223-276, Springer, New York, NY, USA, 1997.

[6] I. Podlubny, Fractional Differential Equations, Academic Press, San Diego, CA, USA, 1999.

[7] R. Gorenflo, A. A. Kilbas, F. Mainardi, and S. Rogosin, MittagLeffler Functions. Related Topics and Applications, Springer, Berlin, Germany, 2nd edition, 2020.

[8] A. Wiman, "Über den fundamentalsatz in der teorie der funktionen $\mathrm{Ea}(\mathrm{x})$," Acta Mathematica, vol. 29, pp. 191-201, 1905.

[9] N. Alessa, B. Venkateswarlu, P. Thirupathi Reddy, K. Loganathan, and K. Tamilvanan, "A new subclass of analytic functions related to Mittag-Leffler type Poisson distribution series," Journal of Function Spaces, vol. 2021, Article ID 6618163, 7 pages, 2021. 
[10] A. Attiya, "Some applications of Mittag-Leffler function in the unit disk," Univerzitet u Nišu, vol. 30, no. 7, pp. 2075-2081, 2016.

[11] D. Bansal and J. K. Prajapat, "Certain geometric properties of the Mittag-Leffler functions," Complex Variables and Elliptic Equations, vol. 61, no. 3, pp. 338-350, 2016.

[12] B. A. Frasin, "An application of an operator associated with generalized Mittag-Leffler function," Konuralp Journal of Mathematics, vol. 7, pp. 199-202, 2019.

[13] B. A. Frasin, T. Al-Hawary, and F. Yousef, "Some properties of a linear operator involving generalized Mittag-Leffler function," Studia Universitatis Babes-Bolyai Matematica, vol. 65, no. 1, pp. 67-75, 2020.

[14] M. Garg, P. Manohar, and S. L. Kalla, "A Mittag-Leffler-type function of two variables," Integral Transforms and Special Functions, vol. 24, no. 11, pp. 934-944, 2013.

[15] V. Kiryakova, Generalized Fractional Calculus and Applications, Longman Scientific and Technical, Copublished in the United States with John Wiley and Sons, New York, Harlow, 1994.

[16] K. A. Selvakumaran, H. A. AL-Kharsani, D. Baleanu, S. D. Purohi, and K. S. Nisar, "Inclusion relationships for some subclasses of analytic functions associated with generalized Bessel functions," Journal of Computational Analysis and Applications, vol. 24, pp. 81-90, 2018.

[17] F. M. Al-Oboudi, "On univalent functions defined by a generalized Sălăgean operator," International Journal of Mathematics and Mathematical Sciences, vol. 2004, no. 27, 1436 pages, 2004.

[18] G. S. Salagean, "Subclasses of univalent functions," in Complex Analysis - Fifth Romanian-Finnish Seminar. Lecture Notes in Mathematics, vol 1013, C. A. Cazacu, N. Boboc, M. Jurchescu, and I. Suciu, Eds., pp. 362-372, Springer, Berlin, Heidelberg, 1983.

[19] H. M. Srivastava, B. A. Frasin, and V. Pescar, "Univalence of integral operators involving Mittag-Leffler functions," Applied Mathematics \& Information Sciences, vol. 11, no. 3, pp. 635641, 2017.

[20] A. W. Goodman, "On uniformly convex functions," Annales Polonici Mathematici, vol. 56, no. 1, pp. 87-92, 1991.

[21] A. W. Goodman, "On uniformly starlike functions," Journal of Mathematical Analysis and Applications, vol. 155, no. 2, pp. 364-370, 1991.

[22] S. Kanas and H. M. Srivastava, "Linear operators associated withk-uniformly convex functions," Integral Transforms and Special Functions, vol. 9, no. 2, pp. 121-132, 2000.

[23] W. Ma and D. Minda, "Uniformly convex functions," Annales Polonici Mathematici, vol. 57, no. 2, pp. 165-175, 1992.

[24] F. Ronning, "Uniformly convex functions and a corresponding class of starlike functions," Proceedings of the American Mathematical Society, vol. 118, no. 1, pp. 189-196, 1993.

[25] M. P. Santosh, N. I. Rajkumar, P. Thirupathi Reddy, and B. Venkateswarlu, "A new subclass of analytic functions defined by linear operator," Advances in Mathematics: Scientific Journal, vol. 9, no. 1, pp. 205-217, 2020.

[26] E. Aqlan, J. M. Jahangiri, and S. R. Kulkarni, "New classes of k uniformly convex and starlike functions," Tamkang Journal of Mathematics, vol. 35, no. 3, pp. 261-266, 2004.

[27] W. Ma and D. Minda, "A unified treatment of some special classes of univalent functions," Proceedings of the Conference on Complex Analysis (Tianjin, Peoples Republic of China; June
19-23, 1992), Z. Li, F. Ren, L. Yang, and S. Zhang, Eds., , pp. 157-169, International Press, Cambridge, Massachusetts, 1994.

[28] H. Silverman, "Partial sums of starlike and convex functions," Journal of Mathematical Analysis and Applications, vol. 209, no. 1, pp. 221-227, 1997.

[29] E. M. Silvia, "Partial sums of convex functions of order R," Houston Journal of Mathematics, vol. 11, no. 3, pp. 397-404, 1985. 